\title{
VICENTE ORDOÑEZ ROIG ${ }^{1}$
}

\section{El cuerpo del delirio: Antígona, Zambrano, Femen}

\author{
Corpus Delirii: Antigone, Zambrano, Femen
}

\section{RESUMEN}

Investigar el nexo causal que existe entre el cuerpo entendido como espacio de resistencia política feminista y la paralización y asimilación de ese cuerpo a través de un entramado normativo y clínico que acusa de «locas», «perturbadas» o «delirantes» a quienes se posicionan contra los distintos dispositivos de poder es el objetivo principal de este artículo. En primer lugar se estudia la reacción simbólica de Antígona y el veredicto de locura que recae sobre ella, antesala de su posterior sentencia a muerte. A continuación, el intento por reintegrar la locura y el delirio a la vida consciente del ser humano tal y como aparece en la obra de María Zambrano. Por último, la lucha cuerpo a cuerpo de Femen, la reacción tanto de las instituciones como del poder político y la categorización de su actitud como delirante. Palabras clave: locura, normatividad, feminismo, género, poder.

\section{Abstract}

The aim of this paper is to analyze the causality between the body, understood as a space of feminist political resistance, and the suspension and assimilation of that body through a normative and clinical framework that accuses of «mad», «disturbed» or «delirious» those who take a stand against the different devices of power. First of all, I study the symbolic reaction of Antigone, the verdict of madness and her subsequent death sentence. Next, the attempt to reintegrate madness and delirium into the conscious life of the human being as formulated by María Zambrano. Finally, Femen's bodily struggle, the reaction of institutions and political power and the denouncement of madness that falls on them.

Keywords: Insanity, normativity, feminism, gender, power.

\section{SUMARIO}

1.- Introducción. 2.- Entrañas. 3.- Piedad. 4.- Corpus delirii. 5.- Locura. 6.- Les Antigones. -Conclusiones. -Referencias bibliográficas.

\section{Introducción}

La libertad objetiva, las leyes de la libertad real exigen la rebelión de la voluntad contingente: non serviam. Así, volviendo del revés la sentencia con la que Hegel concluye sus Lecciones sobre la filosofía de la historia universal, quisiera iniciar estas 
reflexiones sobre el cuerpo como sujeto político y sobre una de las estrategias de las que se ha servido el statu quo para producir, desubjetivizar e instrumentalizar ese mismo cuerpo: la que pone a su servicio la legalidad jurídico-política y la psiquiatrización disciplinaria. Esta doble vía normativa hace posible la construcción de la categoría de lo corporal como algo uniforme y estable, incomunicado, cerrado sobre sí y circular, suprimiendo las superficies laminares que lo conforman y desplazando toda esa amalgama de flujos, relaciones y compuestos, fibras conjuntivas, caudales y ramificaciones que forman parte de su estructura interna. Una vez prefijado, una vez situado y construido un esquema corporal determinado, resulta sencillo patologizar al cuerpo o a los cuerpos que no se ajustan a ese modelo objetivo. Estas consideraciones me llevan a pensar en un arquetipo como primer polo de resistencia contra las aspiraciones totalitarias y los reflejos condicionados arbitrarios que imponen sobre el cuerpo una categorización tan restrictiva: el cuerpo de Antígona, la joven que levanta su voz contra Creonte, tirano de Tebas en el que se concentran los rasgos fenotípicos y psicológicos que encontramos en personajes históricos como Hierón, Calvino o Franco -megalomanía, exigencia de obediencia y subordinación, violencia descarnada y sumisión a la norma, cualquiera que esta sea. Utilizaré los torsos desnudos de Femen y sus tácticas de acción directa como segundo polo de mi argumentación para estudiar cómo la Realpolitik desvirtúa a sus activistas acusándolas, entre otras cosas, de desplegar acciones desordenadas y punibles que pertenecen al ámbito de la demencia y la locura ${ }^{2}$. Entre esos dos polos situaré, como un cable subterráneo que se extiende entre las costas de dos continentes lejanos, el pensamiento de María Zambrano, sobre todo en lo que este tiene de corporal, a saber: la materialidad de las entrañas, el concepto de piedad y su misma experiencia delirante. Por último, me aproximaré críticamente a la figura de Antígona analizando la reapropiación que ha hecho de ella el movimiento francés Les Antigones.

\section{Entrañas}

Tras la ruina y hundimiento de las dos naves en las que trascurre su vida, la política y la genética, Antígona, sola en la inmensidad de un océano desconocido, decide quitarse la vida ante la perspectiva de ahogarse lentamente como el náufrago que perece tras el resquebrajamiento de su barco. ¿O no fue exactamente así? ¿Puede reducirse un personaje tan complejo y rico en matices psicológicos a la muerte voluntaria? ¿No hay algo en Antígona que se resiste a ser conceptualizado de esa forma? Posiblemente. La joven virgen se encuentra en un espacio de pura

2 No dispongo de espacio en estas breves consideraciones para analizar, de un lado, el contenido teórico de la propuesta de Femen; de otro, la recepción que han tenido sus acciones en los estudios feministas. Consúltese al respecto los artículos de O'Keefe (2014), Khrebtan-Hörhager y Kononenko (2015), Reverter Bañón (2016), Dias Martins (2016) y Caviedes (2017) y los ensayos de Zychowicz (2016), Timm y Sanborn (2016) y Goujon (2017). Asimismo, cfr. los documentales Nos seins, nos armes (2013) dirigido por Nadia El Fani y Caroline Fourest y Ukraine is not a Brothel (2013) de la directora australiana Kitty Green, que registran los primeros pasos de Femen y analizan el papel que juega en la organización Viktor Sviatsky. 
indeterminación que escapa por momentos a los dominios de la lógica y la razón. Quizá por ello Sófocles trata de apresar a Antígona con términos como disboulían, anoos o morós, conceptos todos que pertenecen al campo semántico de la locura. Zambrano recurre a otros conceptos que solo tentativamente explican lo que Antígona sufre y padece. Uno de ellos es el de «entraña» y la red léxica de significantes que en castellano tienen un rasgo semántico común: «entrañar», «entrañamiento» o «desentrañar». «Entraña» viene del latín interanea (intestinos), forma neutral y plural usada como sustantivo del adjetivo latino interaneus. A su vez, el adjetivo interaneus se forma sobre el adjetivo latino interus (de dentro). En un primer nivel, por tanto, «entraña» remite al organismo interior de Antígona, a ese pliegue somático de muy difícil acceso que requiere de una intensa disciplina fenomenológica para descifrar lo que es o está ahí. Las entrañas conectan, además, con el elemento ctónico que recorre la obra de Sófocles: hay que devolver a la tierra lo que es suyo, restituirla de sus muertos, cultivar las profundidades del inframundo.

Para Zambrano las entrañas son, no solo aquello interior y muy remoto, sino y sobre todo la sede del padecer, esto es, el lugar que simboliza la capacidad humana de experimentar, sufrir y soportar la vida en toda su amplitud. «Sentir la multiplicidad, la discordancia, lo heterogéneo [...], sentir lo que no se dice, estar condenado al silencio. ¿Podrá la razón hablar por todo esto?» (Zambrano, 2011: 223). La razón es condición necesaria, no suficiente, para aprehender la vida en su compleja red de interrelaciones. Sófocles lo sabe. El prólogo de la tragedia, con unos versos iniciales de muy difícil traducción, pone de manifiesto que hay haces de luz en la trama de los Labdácidas que, como los destellos de un faro, solo son percibidos por la razón de manera intermitente o discontinua ${ }^{3}$. Antígona, que sufre en su pequeño cuerpo la violencia dogmática de Creonte, tiene en sus entrañas su enigma (Zambrano, 2011: 1134).

«Entraña», que guarda una proximidad tanto léxica como semántica con inventro, uno de esos neologismos a los que recurre Dante para dar cuenta de su experiencia extática en el Paraíso (XXI, v. 84), es una metáfora que capta adecuadamente la corporeidad de Antígona, sus llantos y súplicas, su padecimiento, las acusaciones de locura, los gritos mudos y solitarios o la angustia de su destino y el de los suyos. La heroína de Sófocles, que ni puede enterrar a Polinices ni tan siquiera llorarlo porque Creonte lo ha prohibido bajo amenaza de muerte, experimenta muy hondo el desajuste entre su deseo de justicia y la arbitrariedad de la ley del tirano. Además, y por un terrible juego de espejos del que parece dar cuenta Saint-Saëns en la música de su Antígona, la joven es condenada a morir en vida sepultada en las entrañas de piedra de una tumba invisible para los mortales: «la enterraré viva en una gruta

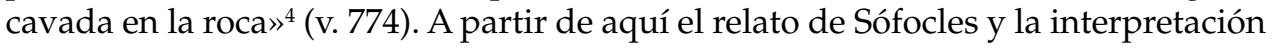

3 El primer hexámetro de la tragedia, o koinon autádelphon Isménes kára (¡oh Ismene, hermana entre hermanas, de mi misma sangre!), se pliega en una multitud de planos hermenéuticos. Steiner lo ha examinado con rigor filológico (Steiner, 1987: 163 y ss.). La traducción de Hölderlin Gemeinsamschwesterliches! o Ismenes Haupt !- quizá sea uno de los intentos más precisos por verter a una lengua moderna la palabra de Sófocles (Hölderlin, 1959: 223).

4 La traducción de los fragmentos de la obra de Sófocles, así como las traducciones de los manifiestos, artículos y ensayos no publicados en castellano o catalán son mías. 
de Zambrano, como los senderos del jardín de Borges, se bifurcan. Para Zambrano, Antígona no puede cometer un acto tan desesperado y violento como el suicidio porque no ha tenido tiempo de explorar esa posibilidad vital. La joven tebana es una sombra, una ensoñación o, más bien, una crisálida inmovilizada en el lapso que va de su estado larvario a la mariposa que es en potencia:

[Antígona] es la primavera de la conciencia humana, la pureza de la conciencia y por ello resurgirá una y otra vez de su sepulcro para alumbrar el mundo. Y reaparecerá siempre en forma de muchacha que no ha tenido tiempo de pensar en sí misma, cegada por el amor sin mancha; es decir, por la Piedad (Zambrano, 2014: 292).

El que Antígona gane el horizonte de una nueva y desconocida conciencia viene precedido de dos momentos dramáticos: la espantosa soledad a la que se ve lanzada y la acusación de locura que recae sobre ella. Creonte quiere construir un túmulo de piedra y esconder en su centro a Antígona para que muera por inanición. Al ser forzada a vivir su propia muerte, la joven labdácida es arrastrada hacia lo inescrutable, hacia lo totalmente insondable, a saber: hacia un lugar en el que ninguna palabra es válida ni ninguna definición posible. Zambrano le hace decir a su Antígona: «estoy aquí, en las entrañas de piedra, ahora lo sé, condenada a que nada nazca de mí. Virgen era, me trajeron no a la tierra, a las piedras, para que de mí ni viva ni muerta nazca nada. Pero yo estoy aquí delirando, tengo voz, tengo voz» (Zambrano, 2011: 1135). Antígona delira pero conserva la voz en su delirio solitario, una voz interior material muy próxima al grito angustiado o al eco ${ }^{5}$.

No carece de importancia que Antígona conserve la voz en el drama de Zambrano. En su conferencia «La voz pública de las mujeres», Mary Beard (2018) ha puesto de manifiesto que el método de silenciamiento de la mujer ${ }^{6}$ en las sociedades patriarcales es el procedimiento por el que se ajusta su discurso al espacio privado, como Telémaco hizo con Penélope al obligarla a callar ${ }^{7}$ (Beard, 2018: 16). La condición de posibilidad de la existencia de una nueva escala de valores y un modelo normativo diferente pasa porque la voz de Antígona no se deje silenciar: a una moral viril cimentada en la obediencia ciega, la sumisión jerárquica y el egoís-

5 Adriana Cavarero ha estudiado en profundidad la intrahistoria de la voz. La voz es una invocación que aparece por vez primera cuando «los lazos intrauterinos -que son rítmicos, musicales- por los que la madre está ligada a la recién nacida se rompen» (Cavarero, 2005: 169). Sobre el simbolismo de la voz de Antígona, cfr. (Butler, 2000: 58).

6 Empleo las categorías binarias mujer/hombre, femenino/masculino con reservas, principalmente porque corro el riesgo de que esas categorías se transformen en un instrumento de las estructuras de poder contra las que llamo la atención. En todo caso coincido con el juicio de Judith Butler: «no hay ninguna posición política purificada de poder, y quizá sea esa impureza lo que ocasiona la capacidad de acción como interrupción eventual y cambio total de los regímenes reguladores» (Butler, 2007: 32). Para una revisión del problema del género y de la construcción de la categoría gramatical «mujer» en la estructura heterosexual dominante, cfr. Sørensen (2000), Alvanoudi (2014) y Ehrlich, Meyerhoff y Holmes (2017).

7 Para un desarrollo de este argumento, así como del recurso al mansplaining como imperativo oclusivo de la comunicación, cfr. (Ferrer-Alcantud, 2017: 154). 
mo utilitarista se opone una praxis fundada en el respeto, el coraje y la resolución encarnada por la joven tebana. Su voz es, por ello, la revelación a través de la cual, en un momento fugaz, se abre paso una insólita areté: el habitante de la ciudad debe ser ante todo una persona consciente que, por medio de la solidaridad o, más exactamente, de lo que Sófocles denomina «amor compartido» (synphilein v. 523), alumbre una ética de la cooperación y la ayuda mutua.

\section{Piedad}

Antígona, que en su mismo nombre lleva su secreto-Anti-gone, la que no puede dar a luz, aquella que se opone o es contraria a la generación- rompe con la hamartía o maldición de los Labdácidas gracias a su acción piadosa. Por eso Zambrano afirma que en Antígona conciencia y piedad son lo mismo (Zambrano, 2014: 292). Ahora bien, ¿qué entiende exactamente Zambrano por piedad? Nos encontramos ante otro de los conceptos clave de su pensamiento, que estudia en profundidad en el segundo capítulo de un libro fundamental en la producción de la autora malagueña: El hombre y lo divino. En un primer nivel, la piedad es ese amor afectuoso que se ofrece de forma gratuita, como un don, a los padres y seres queridos. Mas también la piedad apunta a esa acción que hace que los humanos sean capaces de tratar adecuadamente con el ámbito divino. No obstante, quedarse en este segundo nivel sería un error en opinión de Zambrano. ¿Por qué? ¿Acaso la piedad no inspira, por amor a los dioses, tierna devoción a las cosas sagradas y, por el amor al prójimo, actos de amor y compasión? Ciertamente. Sin embargo, Zambrano amplía el área de influencia de la piedad:

Cuando hablamos de piedad, siempre se refiere al trato de algo o alguien que no está en nuestro mismo plano vital; un dios, un animal, una planta, un ser humano enfermo o monstruoso, algo invisible, algo que es y no es. Es decir, una realidad perteneciente a otra región o plano del ser en que estamos los seres humanos, o una realidad que linda o está más allá de los linderos del ser (Zambrano, 2011: 227).

La piedad cobra un nuevo estatuto ontológico en Zambrano, que la desliga de la sacralidad para acercarla a lo deforme, enfermo o monstruoso, a aquello que se sitúa en los límites de la razón y que el entendimiento percibe de forma borrosa ${ }^{8}$. Al mismo tiempo, con esta idea Zambrano recupera lo que la piedad tiene de acción, relación o sentimiento, alejándose también de lo que la filosofía, al menos desde Platón, ha entendido por piedad: una virtud o modo de ser del hombre justo (Euthyphro 11e). Entonces, ¿qué cabe entender por piedad? ¿Es posible decir o mostrar qué es o solo podemos aproximarnos a ella tentativamente y casi siempre por vía apofántica o negativa? Lo que descubrimos al escrutar la piedad, según Zambrano, es una acción que permite entender lo diferente, la heterogeneidad, los

8 En la idea de piedad de Zambrano reverbera el concepto de lo numinoso como mysterium tremendum desarrollado por Rudolf Otto en su obra Das Heilige (Otto, 1917: 28); i.e.: una experiencia no-racional que remite a una realidad incognoscible subyacente en todas las cosas. 
distintos planos en los que se articula la realidad o lo que se muestra radicalmente como lo otro de sí (Zambrano, 2012: 69). Aunque para la tradición filosófica, desde Descartes en adelante, la realidad pertenece exclusivamente a la razón objetiva y al saber cuantificable y demostrable empíricamente, para Zambrano la realidad no se agota en las ideas claras y distintas con las que la concebimos: la realidad está compuesta de un tejido poroso por el que se cuelan elementos extraños a la pura razón que hacen que ese devenir-fou al que alude Deleuze se sienta como una presencia manifiesta (Deleuze, 1969: 17).

Desde el punto de vista de Zambrano, el arte ha entendido mejor que el pensamiento racional esa realidad liminar en la que conviven lo deforme y lo esférico, lo enfermo y lo vigoroso, lo monstruoso y lo apolíneo (Zambrano, 2011: 223). En nombre de la racionalidad se ha tratado de apartar $y$, cuando ha sido posible, arrancar esas zonas oscuras que escapan a su dominio: de los leprocomios a las naves de los locos, pasando por el positivismo criminológico o la psicocirugía prefrontal, los episodios en los que se intenta extirpar todo aquello que cae del otro lado de la razón están debidamente documentados (Foucault, 1998; Pietikainen, 2015; Scull, 2015). En este punto hay que subrayar que el pensamiento racional ha empleado la locura para justificar sus técnicas de sometimiento de los cuerpos y, junto a los degenerados, enfermos venéreos o blasfemos, se ha recluido desde antiguo a mujeres en sanatorios psiquiátricos por mor de su corporeidad mórbida y su comportamiento demente. El ensayo de Phyllis Chasler Women and Madness (1972) es, quizá, el primer intento por demostrar críticamente que los juicios sobre la salud mental, las terapias y tratamientos y las definiciones de curación son necesariamente androcéntricos. Que la clínica tenga la capacidad de determinar si una mujer está 'cuerda', es 'neurótica' o 'bipolar,' depende de una ética masculina de la psiquiatría basada en suposiciones invisibles pero explícitas de sociedades heterosexuales fuertemente correctoras. Por eso, a finales de siglo XX Andrea Dworkin pudo decir que resistirse a las formas de control masculinas lleva «a la violación, la agresión, la indigencia, el aislamiento o el exilio, el confinamiento en una institución mental, la cárcel o la muerte» (Dworkin, 1978: 15). Por su parte, Zambrano considerará que a las mujeres que se posicionan contra el muro de contención del poder les espera invariablemente la celda o la hoguera: no hay piedad para ellas ${ }^{9}$ (Zambrano, 2014: 302).

Sobre la Antígona de Sófocles y la de Zambrano, pero también sobre las activistas de grupos feministas que, como Femen, luchan abiertamente contra esas mismas instituciones patriarcales, se deja caer la acusación de locura. La sociedad biopolítica disciplinaria a la que se refiere Foucault (2009: 268) no solo produce fuerzas, las hace crecer y las ordena, también interviene, controla y disciplina esos cuerpos por medio de la coacción heteronormativa y la vigilancia médica ${ }^{10}$. En última instancia, que el cuerpo de la mujer pueda ser entendido como una cinta de Möbius en la que razón y locura se pliegan, superponen y entrelazan, permite abstraerlo, objetivarlo y someterlo a una práctica continuada de dominación.

9 Sobre el pensamiento político de Zambrano, cfr. (Urdanibia, 1994: 110 y ss.).

10 Para un análisis del horizonte abierto del biopoder, cfr. (Landaeta Mardones, Arias Krause y Espinoza Lolas, 2017: 100). 


\section{Corpus delirii}

La acción piadosa de Antígona apura el conflicto trágico y muestra in nuce el origen de una nueva conciencia y con ella el nacimiento de una inédita libertad subjetiva. ¿Y cómo podrían las acciones de Antígona no abrir posibilidades inexploradas al ser humano si ya no deja que la violencia ni el miedo al tirano la dominen ${ }^{11}$ ? Antígona lleva en sí la negación, y esta actúa como freno epistemológico de todo dogma y pretensión autoritaria. La última heroína de la saga de Layo no es solo el «punto atómico de la conciencia» como pretendía Hegel (1952: 422); es eso y algo más: una mujer que, atrapada en el entramado coercitivo del poder político e institucional, le hace frente con la sola fuerza de su corporeidad adolescente.

La correa de transmisión que une los engranajes de la maquinaria violenta del tirano se rompe con la actitud desafiante de Antígona, que cargará por ello con la acusación de locura primero, con su propia muerte después. Ahora bien, ¿no representa Antígona la misma lucidez? Pero si Antígona es la imagen nítida de la lucidez, ¿de qué depende la lucidez de su conciencia? ¿No es acaso de su doble, esto es, de la manía, del delirio ${ }^{12}$ ? Ese vaivén entre cordura y locura está presente en la tragedia de Sófocles como una espada que atraviesa, de dentro a fuera, el corazón sufriente de Antígona. Aparentemente, delirio y lucidez, locura y razón, son como asíntotas que no pueden cruzarse si no es en el infinito. El delirio de Antígona, sin embargo, es una especie de paso atrás, una epojé con la que contrapesar to deinótaton, aquello tan terrible que aguarda latente en el anverso de la conciencia ${ }^{13}$

11 En la obra de Irigaray (1993), pero también en la de Honnig (2013) y Taxidou (2107) pueden encontrarse los cimientos de una interpretación agonística en clave feminista de la sororidad o hermandad de mujeres que hace posible Antígona.

12 Hay que señalar, no obstante, que el radical indoeuropeo del campo semántico «manía» es mnyo-, forma sufijo de la raíz men-, pensar. El lingüista francés Pierre Chantraiine argumenta que el verbo griego «enloquecer» (mainomai) se ha disociado de la noción general de «pensar» para aplicarse a la noción de «ardor loco» y «furioso» (Chantraiine, 1968, 658). Aun así, ¿no es ya la locura una variante $\mathrm{y}$, sobre todo, una posibilidad lógica del pensamiento, y viceversa?

13 Conocido es el análisis de Heidegger de estos versos de Antígona (Heidegger, 1987: 136 y ss.). No obstante, su exégesis está influenciada decisivamente por lo que, según Zambrano, palpita en el proyecto metafísico occidental: «la metafísica europea es hija de la desconfianza, del recelo y en lugar de mirar hacia las cosas, en torno de preguntar por el ser de las cosas, se vuelve sobre sí en un momento distanciador que es la duda [...]. La razón se afirmaba cerrándose y después, naturalmente ya no podía encontrar otra cosa que a sí misma. De ahí la angustia» (Zambrano, 2015: 747-748). La angustia parece ser la raíz originaria de la metafísica, pero solo parece ser: en la metafísica griega -y Antígona supone, entre otras cosas, una síntesis del pensamiento metafísico griego- la transparencia, la luz y la esperanza conviven con las tinieblas que se acumulan en las galerías de la razón. Sófocles, no hay duda, anticipa en el primer canto del coro que nada hay tan terrible como el ser humano. Sin embargo, añade que poseído como está de una destreza sinigual para inventar nuevas posibilidades de vida, el sapiens se deja llevar unas veces por el mal, otras por lo esthlós, i.e., por lo que es noble y moralmente bueno (v. 366). La palabra de Sófocles nombra por tanto la alienación y la violencia y alerta sobre los peligros inherentes a toda acción humana. No obstante, enumera también las posibilidades de una vida diferente. Esa dualidad moral está inscrita en el proyecto existencial del hombre griego como las palabras fuck y morals están pintadas en los torsos desnudos de las activistas de Femen. Privilegiar uno sobre otro como hace Heidegger es erróneo: se trata de trayectos contrapuestos que no son sino sendas de un mismo camino. 
(v. 334). En Zambrano también se produce una metabolización del delirio o, más bien, de los delirios, a los que integra en su propia vida y plasma en sus escritos. Los delirios representan para la pensadora malagueña la contrapartida o lado opuesto de una subjetividad múltiple que se cuestiona a sí misma y se reinventa, y nada tienen que ver, por tanto, con los trastornos cerebrales transitorios y los cuadros descriptivos de la psicología clínica: «es el propio ser el que se manifiesta en el delirio, el ser no vivido, no vivido, la posibilidad. Eso es el delirio, una posibilidad» (Zambrano, 2014: 287).

La locura de Antígona, sin embargo, no se representa en escena como una posibilidad del coraje sino como una desviación física de la razón. Ya Ismene le advierte en el proemio de lo insensato de su conducta y el corifeo canta en la segunda antistrofa que Antígona ha cometido una aphrosyne, un disparate (v. 383). Efectivamente, los programas clínicos se han desarrollado a menudo gracias a que el cuerpo de la mujer es analizado e integrado en el campo de las prácticas médicas en virtud de una patología que le es intrínseca: su sexualidad desbordante (Foucault, 1998: 127). El renombrado alienista Jean Étienne Dominique Esquirol, tras realizar en 1817 la autopsia a la revolucionaria Théroigne de Méricourt, fija los parámetros de patologización de las activistas feministas a partir de un esquema triple: desacredita sus acciones (son peligrosas, irresponsables y deplorables), híper-sexualiza a las militantes (las llama ninfómanas, prostitutas, locas por su cuerpo y por su útero) y especializa su locura (las hay histéricas, como también monomaníacas ambiciosas o locas lúcidas ${ }^{14}$ ) (Ripa, 2017). La idea que articula Esquirol tiene, como mínimo, dos estratos o sedimentos: en un primer nivel, leemos que las mujeres que cuestionan el poder constitutivo están privadas del juicio o del uso de la razón. Sin embargo, al profundizar en su argumento, puede percibirse nítidamente la sentencia que afirma que, ontológicamente, la categoría 'mujer' es una desviación del estado de equilibrio ${ }^{15}$.

Hoy no es difícil comprobar cómo se trata de aplazar las demandas y aplacar a las activistas feministas que se enfrentan al statu quo a través de unos programas preceptivos que tienen en la histerización del cuerpo a su pièce de résistance. Esta es, no por casualidad, una de las estrategias que se ha seguido contra Femen. Pero, ¿qué o quiénes son Femen? En 2008 tres jóvenes ucranianas -Anna Hutsol, Inna Shevchenko y Oksana Sashko- forman en Khmelnytskyi una célula feminista organizada al modo de los grupos de afinidad libertarios para protestar contra el turismo sexual de Ucrania en particular y contra el trasfondo de la siniestra industria del porno en general (Venner, 2017). Desde entonces, Femen ha ganado notoriedad debido, en parte, a que sus acciones han desbordado el ámbito nacional para

14 La experiencia psiquiátrica de Sulamith Firestone, narrada en tercera persona en Airless Spaces, constituye un hito en la descripción de las técnicas de normalización clínica desde una perspectiva feminista: «estaba lúcida, sí, pero a qué precio. A veces reconocía en los rostros de los otros la alegría y la ambición y otras emociones que recordaba haber tenido alguna vez, hace mucho tiempo. Pero su vida estaba arruinada, y carecía de plan de rescate» (Firestone, 1998: 59).

15 Ya en el siglo XX Simone de Beauvoir escribe que de su persona se han forjado dos imágenes: de un lado, la de loca (folle), medio loca (demi-folle) o excéntrica; de otra, la de institutriz. «Lo esencial es presentarme como una anormal» (Beauvoir, 1980: 624). 
convertirse en un vector discursivo que reflexiona críticamente sobre la ubicuidad de las estructuras patriarcales, la hipostatización de lo masculino o la homofobia a través de formas de resistencia corporal (Khrebtan-Hörhager y Kononenko, 2015). En su manifiesto escriben:

Es necesario que revuelvas tu cuerpo contra la injusticia, que movilices cada una de sus células en la guerra contra el patriarcado y la humillación, y que digas al mundo:

¡Nuestro dios es mujer!

¡Nuestra misión es protestar!

¡Nuestras armas son nuestros pechos desnudos!

Así nace Femen y comienza el sextremismo (Femen, 2014: 5).

Las activistas de Femen consideran que las instituciones religiosas, los Estados autoritarios y la industria sexual han ido tejiendo sobre el cuerpo de la mujer una camisa de fuerza vaporosa que la envuelve y paraliza: «el cuerpo se le ha arrebatado a la mujer y ha sido objeto de una explotación patriarcal monstruosa. El control absoluto del cuerpo de la mujer es el principal instrumento con que se la oprime [...]. Que la mujer proclame que es dueña de su propio cuerpo es el primer paso y el más importante para que consiga la libertad» (Femen, 2014: 6). El que expresen con sus pechos desnudos el conflicto que opera entre corporeidad, de un lado, y estructuras políticas falogocéntricas de otro, constituye la forma visible de su rechazo a la sumisión, accesibilidad y disponibilidad de sus cuerpos. Sus acciones en la cumbre de Davos, en iglesias, prostíbulos y mezquitas, en la feria comercial de Hannover o en el Gran Premio de Automovilismo de Montreal son la consecuencia lógica de ese odio no disimulado contra el orden establecido con las que, a su vez, amplían el campo hermenéutico de los estudios de género.

\section{Locura}

En este punto parece pertinente preguntarse cómo han reaccionado las instituciones a las que Femen se enfrenta en un conflicto permanente desde hace diez años. Portales católicos, movimientos musulmanes y medios de comunicación de distinto signo han mostrado su consternación y repulsa frente a las acciones directas de Femen. En 2014 unas activistas españolas lanzaron bragas pintadas de rojo a Rouco Varela, arzobispo de Madrid. En el blog de Infovaticana se denuncia el hecho con un editorial titulado «FEMEN: el timo de la juventud superficial y el control mental». En el artículo se tilda a las activistas de marionetas y se afirma que están endemoniadas: «creo que detrás de ellas está el demonio, pero se trata de un demonio de carne y hueso, encarnado en hombres que quieren arrancar a las mujeres su condición más auténtica y sagrada: la de ser madres» ${ }^{16}$. Asimismo, New English Review, una revista norteamericana conservadora que ha estado estrecha-

16 Disponible en: https://infovaticana.com/blogs/reflexiones-candil/femen-el-timo-de-la-juventudsuperficial-y-el-control-mental/ (Fecha de consulta: 28/02/2018). 
mente involucrada en el movimiento de la Contrajihad participando en la creación de Stop Islamization of America en 2009, publicó en 2014 el artículo firmado por Enza Ferreri «Radical Feminists' War on Churches». La autora compara arbitrariamente a los musulmanes y a las activistas feministas y los colectivos homosexuales en general porque su objetivo es uno y el mismo: destruir la decencia y moralidad de la civilización cristiana. Más adelante escribe:

El día de Navidad es la época del año favorita de los musulmanes para llevar a cabo ataques contra los cristianos en Oriente Medio, y sus imitadores occidentales parecen tener la misma predisposición. Una activista del grupo feminista Femen, Josephine Witt, interrumpió la misa navideña de la Catedral Mayor de San Pedro, en Colonia [...]. Desnuda, subió al altar gritando 'yo soy Dios', palabras que tenía pintadas en su cuerpo. La belleza de la Misa de Navidad alterada -siquiera por unos momentos- by the beast of deluded madness, por la bestia de la locura alucinada $^{17}$.

Entre los sectores más conservadores del sur de Alemania la acción directa de Femen causó estupor. El diario de Colonia Express publicó en portada el siguiente artículo: «Después del ataque en topless [el cardenal] Meisner dice de la activista de Femen: 'es una pobre mujer enferma'». Y prosigue con unas declaraciones del entonces cardenal: «todos merecen la bendición, incluso la mujer perturbada (verwirrte) de antes» ${ }^{18}$. Es posible que el Express se sintiera también atacado, directa o indirectamente: su edición digital dedica toda una sección a la pornografía, uno de los objetivos de Femen. En otro artículo del Hufftington Post titulado «Femen: Please Slow your Roll», Vlad Chituc informa del International Topless Jihad Day organizado por Femen en abril de 2013. Amina Tyler, activista tunecina del grupo, pintó la palabra Femen junto al muro de un cementerio de la ciudad de Kairouan donde el grupo salafista Ansar Sharía había convocado un acto (Chiappelli, 2016). Fue detenida por ello: «los informes revelan que Amina fue internada en un hospital psiquiátrico, y muchas figuras islámicas de alto rango han pedido una respuesta de extrema violencia a sus fotos» ${ }^{19}$.

El establishment político también se ha visto contrariado por las acciones de Femen. En muchos casos la respuesta ha sido puramente judicial y se ha denunciado a sus activistas por conducta indecente, actos dolosos o exhibicionismo. En otras ocasiones, sin embargo, se las ha atacado, bien en primera persona, bien a través de medios de comunicación afines, aludiendo a su comportamiento delirante. En Francia, que Inna Shevchenko inspirara la imagen de Marianne en los sellos oficiales del país, símbolo y encarnación de la República, provocó airadas reacciones. La revista digital 24heuresactu.com relata con indignación: «mientras Kiev espera más

17 Disponible en: http://www.newenglishreview.org/custpage.cfm/frm/160329/sec_id/160329 (Fecha de consulta 03/03/2018).

18 Disponible en: https://www.express.de/koeln/nach-oben-ohne-attacke-meisner-ueber-femenaktivistin---arme--kranke-frau--4071498 (Fecha de consulta 28/02/2018).

19 Disponible en: https://www.huffingtonpost.com/vlad-chituc/femen-please-slow-yourroll_b_3040096.html (Fecha de consulta 28/02/2018). 
que nunca firmar un acuerdo de asociación con la Unión Europea en noviembre de 2013, la diplomacia ucraniana ve la llegada de hordes de folles de pechos desnudos que no tienen nada más que odio a su patria». Además de locas, las llama histéricas y llega a compararlas con parásitos transnacionales ${ }^{20}$. Por otra parte, el periodista y escritor ultraconservador Eric Zemmour, próximo a los planteamientos ideológicos más extremistas del Frente Nacional francés, ha utilizado en varias ocasiones el argumento de la histeria, la locura y el delirio para denunciar a Femen en particular y al feminismo en general: según Zemmour, Francia es víctima de una «conspiración lésbico-feminista masónica» ${ }^{21}$. No es de extrañar, quizá, que la misma Marine Le Pen, objetivo recurrente de las Femen, las haya desacreditado retóricamente utilizando distintas estrategias verbales. En abril de 2017, en el transcurso de un mitin en el Zénith de París, la candidata a la presidencia del Frente Nacional era interrumpida, primero por una activista que saltaba al estrado con un ramo de flores; después por otra que, desde la tribuna, gritaba contra la dirigente francesa. Le Pen declaró a continuación: «estas radicales de extrema izquierda marchent sur la tête (actúan de forma irracional)»22.

Pensar que solo el lobby conservador y ultraconservador se ha pronunciado contra las Femen utilizando el argumento de la locura sería un error. Es el caso del periódico alemán Die Zeit, en cuyo consejo de redacción se encuentran personalidades de la política y la cultura germanas ligadas al SPD como Jutta Allmendinger, Helmut Schmidt o Michael Naumann. En una entrevista a Alexandra Shevchenko realizada en 2012, la primera pregunta que se le formula es la siguiente: «Frau Schewtschenko, würden Sie sich selbst als verrückt bezeichnen? (señora Shevchenko, ¿se describiría a sí misma como loca?)». El artículo es provocativo y, ciertamente, Shevchenko juega con el abanico de posibilidades que permite el concepto de locura -la activista declara que el feminismo es una ideología necesaria para todas las mujeres del mundo, y añade: «esto quiere decir que tenemos que luchar, incluso con armas locas» ${ }^{23}$. En todo caso, lo que el periodista de Die Zeit Steffen Dobbert refleja con su pregunta es, no solo la normalización de una narrativa política que ha ido construyendo a lo largo del tiempo un discurso reificante en torno al género, sino la asimilación de ese constructo sociocultural denominado 'cuerpo femenino' a la locura.

\section{Les Antigones}

Los torsos desnudos de Femen pueden funcionar como un catalizador de los procesos políticos, una táctica telegénica espectacular, una provocación insolente contra el aparato estatal, una regurgitación de las leyes sancionadas paternalmente

20 Disponible en: http://24heuresactu.com/2013/09/04/il-fait-si-bon-en-francepour-les-femen/ (Fecha de consulta 27/02/2018).

21 Disponible en: http:/ /www.dailymotion.com/video/x1bj5r2 (Fecha de consulta 27/02/2018).

22 Disponible en: http://www.rtl.fr/actu/politique/video-presidentielle-2017-le-discours-de-marinele-pen-perturbe-par-une-manifestante-7788179546 (Fecha de consulta 26/02/2018).

23 Disponible en: http://www.zeit.de/sport/2012-06/interview-femen-ukraine-protest (Fecha de consulta 03/03/2018). 
o una modalidad de acción directa que utiliza una gramática desafiante para llamar la atención sobre técnicas político-sociales de subordinación de los cuerpos. En todo caso, su puesta en escena parece no dejar indiferente a nadie. En 2013 se da a conocer en Francia un grupo de mujeres autodenominadas Les Antigones después de que una de sus activistas se infiltre y sea entrenada por Femen ${ }^{24}$. Les Antigones lo forman unas cincuenta mujeres que no se adhieren a confesión o partido político alguno ni tampoco se reconocen en lo que para ellas es la ideología minoritaria pero dominante de las teorías de género y el sextremismo:

Si la primera acción de Les Antigones fue una rebelión contra Femen, oponerse a ellas no es nuestra meta [...]. Abogamos por la feminidad de las mujeres: ésta es nuestra naturaleza coherente y profunda. La afirmación es el primer paso para enriquecer a la sociedad con lo mejor de nosotras mismas. Cada una de nosotras lleva en sí la promesa de ser una mujer consumada y comprometida. [...]. Nosotras, Les Antigones, privilegiamos la legitimidad sobre la legalidad. Si las leyes escritas por los hombres anulan las leyes naturales -es decir, las normas no escritas que son la base de la experiencia humana- tenemos el deber de rebelarnos. No permitiremos que la common decency, la sensatez y la dignidad que deben regir las leyes y los acontecimientos de nuestra sociedad queden sepultados ${ }^{25}$.

Esta declaración de principios muestra de forma más o menos evidente el itinerario de Les Antigones. Su objetivo puede resumirse en, al menos, tres puntos: 1) defensa de una posición epistemológica esencialista; 2) rechazo de las políticas implementadas por la entonces ministra de Justicia francesa Christiane Taubira; 3) asunción acrítica de estructuras hegemónicas patriarcales. Dejando de lado el contenido teórico de su propuesta y los objetivos perseguidos, creo que deben señalarse dos errores en su apropiación de algunos conceptos inspirados por la Antígona de Sófocles. En primer lugar, al defender que «las leyes escritas anulan las leyes naturales [y por ello] tenemos el deber de rebelarnos», en referencia a ley francesa que regula las relaciones homosexuales y al proyecto de ley de gestación subrogada, confunden biología y cultura, ciencia e ideología. Por una parte, idealizan la complementariedad heterosexual de los cuerpos y extienden, consciente o inconscientemente, la representación de las relaciones del poder con la sexualidad. De su manifiesto se desprende, además, que entienden el género como una constante cultural inmodificable. ¿Y pueden hoy pasarse por alto los factores endocrinos y genéticos asociados a la homosexualidad en animales y humanos (Bagemihl, 1999; Savic y Lindström, 2008; Balthazart, 2012; Gómez et al., 2017)? En segundo lugar, Les Antigones llevan a cabo una transvaloración completa de los valores al afirmar que el civismo, la sensatez y la dignidad están amenazadas

24 La literatura ha sido más precisa a la hora de proyectar los personajes de la tragedia sobre el papel de calco de la historia, y más plausible es identificar a Antígona con Ulrike Meinhof (Böll, 1992) y a Creonte con George Bush (Heany, 2004), que escuchar la voz magmática de la joven en el proyecto de Les Antigones.

25 «Manifeste des Antigones», en Les Antigones. Disponible en http://lesantigones.fr/manifesteantigones / (Fecha de consulta 19/04/2018). 
principalmente por las teorías de género, lo que las convierte en el enemigo en sentido óntico. Señalar como enemigo a quienes denuncian la fetichización de los órganos sexuales femeninos, transformados en puro valor de cambio (Llamas, 2013: 91); a quienes muestran la anfibología enajenante que determina y regula la identidad (Jaeggi, 2016: 145); a quienes indican que hay que localizar las estrategias de repetición normativa que posibilitan las identidades construidas (Butler, 2007: 286); señalar, en definitiva, como enemigo a las teorías de género equivale paradójicamente a convertir a Antígona en doble, no en contrafigura de Creonte.

\section{Conclusiones}

Con estas reflexiones sobre Antígona, Zambrano y Femen he querido alertar contra los procesos que asignan de manera jerarquizada subjetividades, identidades y formas de comportamiento alienadas en función de diferencias de género artificiales y ambiguas. La locura es un dispositivo corrector que la fuerza hegemónica organiza en su apropiación de la corporeidad femenina, y cualquier movimiento de masas o incluso de pequeños grupos que pretenda realizar la justicia social, alterar los estereotipos de género vigentes o introducir cambios en las instituciones de carácter patriarcal será visto como una amenaza intolerable. En efecto, los múltiples dispositivos de poder, mediante un entramado político-jurídico heteronormativo, buscan castigos ejemplares para quienes desafían su primacía. Los casos en los que se juzga una conducta de «delirante» ocupan así el primer plano. Aunque el poder actual es contingente y forma parte de los antagonismos concretos de nuestras sociedades contemporáneas, quienes lo detentan, siguiendo una tradición multisecular, siguen alimentando el reflejo según el cual la lucha contra las fuerzas propias del dominio en que se ejercen constituye flagrante delito. La resistencia al poder deberá ser, pues, poliédrica, múltiple y sutil, contra la ley y la norma, contra las reglamentaciones y los modelos. Los discursos liberadores deben ser muchos, procedentes de distintos bricolajes, nunca uno, la teoría, fósil del pasado.

\section{REFERENCIAS BIBLIOGRÁFICAS}

Alvanoud, Angeliki (2014). Grammatical Gender in Interaction: Cultural and Cognitive Aspects, Leiden: Brill.

BAgEMiHL, Bruce (1999). Biological exuberance: animal homosexuality and natural diversity, Nueva York: St. Martin's Press.

BalthazART, Jacques (2012). The Biology of Homosexuality, Nueva York: Oxford University Press.

BEARD, Mary (2018). Mujeres y poder. Un manifiesto, Barcelona: Crítica.

BEAUvoIr, Simone (1987). La fuerza de las cosas, Barcelona: Edhasa.

BölL, Heinrich (1992). Der Deutsche Herbst, Colonia: Kiepenheuer \& Witsch.

Butler, Judith (2000). Antigone's Claim: Kinship between Life and Death, Nueva York: Columbia University Press. 
ButLER, Judith (2007). El género en disputa. El feminismo y la subversión de la identidad, Barcelona: Paidós.

Cavarero, Adriana (2005). For More than One Voice: Toward a Philosophy of Vocal Expression, Stanford: Stanford University Press.

Caviedes, Gabriela (2017). «Feminine Features as Political Tools: The Cases of Femen and Women of Liberia», Araucaria. Revista Iberoamericana de Filosofía, Política y Humanidades, No38, pp. 121-139.

Chantrainne, Pierre (1968). Dictionnaire Étymologique de la Langue Grecque. Histoire des Mots, vol. III, París: Éditions Klincksieck, 1974, $2^{a}$ ed.

ChesLer, Phyllis (1972). Women and Madness: When is a Woman Mad and Who is it Who Decides?, Nueva York: Doubleday \& Co.

ChiAppelli, Tiziana (2016). «Feminismos comparados. Un debate poscolonial entre Túnez y Europa sobre el uso del cuerpo desnudo de las mujeres: la experiencia de Amina», en Millcayac. Revista Digital de Ciencias Sociales, Vol. 3, N4, pp. 65-82.

Deleuze, Gilles (1969). Logique du sens, París: Les Éditions de Minuit.

Dias Martins, Ana Margarida (2016). «Pussy Riot, Femen e as Três Marias: Feminismos zangados e o corpo das mulheres em luta», en Cadernos de Literatura Comparada, No35, pp. 369-378.

Dworkin, Andrea (1978). Right-wing Women, Nueva York: Perigee, 1983, $5^{\text {a }}$ ed.

Ehrlich, Susan, Meyerhoff, Miriam y Janet Holmes (2017). The Handbook of Language, Gender and Sexuality, Oxford: Wiley.

FEMEN (2014). En el principio era el cuerpo, Barcelona: Malpaso.

Ferrer-Alcantud, Coré (2017). «Mary Beard y los dioses propicios», en Asparkía, No30, pp. 151-159.

Firestone, Shulamith (1998). Airless Spaces, Nueva York: Semiotext(e).

Foucault, Michel (1998). Historia de la locura en la época clásica vols. I-II, México D.F.: Fondo de Cultura Económica.

Foucault, Michel (2009). Nacimiento de la Biopolitica. Curso del Collège de France (1978-1979), Madrid: Akal.

Gómez, Francisco R., et al. (2017). «Recalled Separation Anxiety in Childhood in Istmo Zapotec Men, Women, and Muxes», en Archives of Sexual Behavior, Vol. 46, $\mathrm{N}^{\mathrm{o}} 1$, pp. 109-117.

Goujon, Olivier (2017). Femen. Histoire d'une trahison, París: Max Milo Éditions.

Heany, Seamus (2004). The Burial at Thebes: Sophocles' Antigone, Londres: Faber \& Faber, 2005, $2^{\mathrm{a}}$ ed.

HeGEL, Georg Wilhelm Friedrich (1952). Phänomenologie des Geistes, Hamburgo: Felix Meiner. HeIdegGer, Martin (1987). Introducción a la metafísica, Barcelona: Gedisa, 2001, 4a reimpresión.

HöLdERLIN, Friedrich (1959). «Antigonä», en Sämtliche Werke, tomo 5, Berlín: Rütten \& Loening.

Honig, Bonnie (2013). Antigone, Interrupted, Cambridge: Cambridge University Press. IrIGARAY, Luce (1993). Sexes and Genealogies, Nueva York: Columbia University Press. JAEGgI, Rahel (2016). Entfremdung. Zur Aktualität eines sozialphilosophischen Problems, Berlín: Suhrkamp. 
Khrebtan-Hörhager, Julia y Iuliia Kononenko (2015). «Of Fighters and Frames: Femen's Corporeality between the Old, the New, the Yellow and the Blue», en Journal of Intercultural Communication Research, Vol. 44, No3, pp. 224-251.

Landaeta Mardones, Patricio, Arias Krause, Juan Ignacio, y Ricardo EsPinoza Lolas (2017). «La 'cuestión urbana': apuntes para un diagrama de la relación gobierno y policía en Michel Foucault», en Recerca. Revista de pensament $i$ anàlisi, No21, pp. 87-105.

Lamas, Marta (2014). Cuerpo, sexo y política, México D.F.: Océano.

O'KeEfe, Theresa (2014). "My body is my manifesto! Slutwalk, FEMEN and femmenist Protest», en Feminist Review, No107, pp. 1-19.

Отто, Rudolph (1917). Das Heilige. Über das Irrationale in der Idee des Göttlichen und sein Verhältnis zum Rationalen. Munich: C.H. Beck, 1987, $4^{\mathrm{a}}$ ed.

Pietikainen, Petteri (2015). Madness: A History, Nueva York: Routledge.

Reverter BAÑón, Sonia (2016). «El feminismo dislocado: el caso FEMEN», en Eikasía, No 70, pp. 159-187.

RIPA, Yannick (2017). «Folie», en BARD, Christine y Sylvie Chaperon (eds.) (2017) Dictionnaire des féministes. France - XVIIIe-XXIe siècle, París: PUF, pp. 277-278.

SAVIC, Ivanka y Per LINDSTRÖM (2008). «PET and MRI show differences in cerebral asymmetry and functional connectivity between homo- and heterosexual subjects», en PNAS, N¹05, pp. 9403-9408.

Scull, Andrew (2015). Madness in Civilization: A Cultural History of Insanity from the Bible to Freud, from the Madhouse to Modern Medicine, Princeton: Princeton University Press.

Sófocles (1946). Antigone, Bremen: Paderborn.

Sørensen, Marie Louise Stig (2000). Gender Archaeology, Cambridge: Polity Press.

SteINER, Georg (1987). Antígonas. Una poética y una filosofía de la lectura, Barcelona: Gedisa.

TAxidou, Olga (2017). «Tragedy: maternity, natality, theatricality», en FISCHER, Tony y Eve Katsouraki (eds.) (2017). Performing Antagonism: Performance and Radical Democracy, Londres: Palgrave Macmillan, pp. 43-60.

Timm, Annete F. y Joshua A. SAnborn (2007). Gender, Sex and the Shaping of Modern Europe: A History from the French Revolution to the Present Day, Londres: Bloomsbury, 2016, $2^{\mathrm{a}}$ ed.

URdANibia, Javier (1994). «La filosofía política de María Zambrano», en Asparkía. Investigació Feminista, N33, pp. 103-120.

Venner, Fiammetta (2017). «Femen» en BARD, Christine y Sylvie CHAPERoN (eds.) (2017) Dictionnaire des féministes. France - XVIIIe-XXIe siècle, París: PUF, pp. 240-242.

Zambrano, María (2011). El hombre y lo divino, en Obras Completas III, Barcelona: Galaxia Gutenberg.

Zambrano, María (2011). La tumba de Antígona, en Obras Completas III, Barcelona: Galaxia Gutenberg.

Zambrano, María (2012). «Para una historia de la piedad», Aurora: Papeles del Seminario María Zambrano, $\mathrm{N}^{\mathrm{o}}$ Extraordinario, pp. 64-72.

Zambrano, María (2014). Escritos autobiográficos. Delirios. Poemas (1928-1990), en Obras Completas VI, Barcelona: Galaxia Gutenberg. 
Zambrano, María (2015). Filosofía y poesía, en Obras Completas I, Barcelona: Galaxia Gutenberg.

Zychowicz, Jessica (2016). «Femen: A Litmus» en Hashamova, Yana, Holmgren, Beth y Mark Lipovetsky (eds.) (2016). Transgressive Women in Modern Russian and East European Cultures: From the Bad to the Blasphemous, Londres: Routledge, pp. 165-181.

Recibido el 3 de marzo de 2018

Aceptado el 8 de mayo de 2018

BIBLID [1132-8231 (2018): 169-184] 